\title{
A trial with 3'-azido-2',3'-dideoxythymidine and human interferon- $\alpha$ in cats naturally infected with feline leukaemia virus
}

\author{
Bianca Stuetzer ${ }^{1}$, Konstanze Brunner ${ }^{1}$, Hans Lutz ${ }^{2}$ and \\ Katrin Hartmann ${ }^{1}$
}

\begin{abstract}
Feline leukaemia virus (FeLV) infection is still one of the leading causes of infection-related deaths in domestic cats. Treatment with various drugs has been attempted, but none has resulted in cure or complete virus elimination. Human interferon- $\alpha 2 a$ (hulFN- $\alpha 2 a$ ) and $3^{\prime}$-azido-2',3'-dideoxythymidine (AZT) have been proven to decrease antigenaemia in cats infected experimentally with FeLV. The purpose of this study was to assess the efficacy of hulFN- $\alpha 2 a, A Z T$ and a combination of both drugs in cats infected naturally with FeLV in a placebo-controlled double-blinded trial. Fourty-four FeLV-infected cats in which free FeLV p27 antigen was detected in serum by enzyme-linked immunosorbent assay were included in the study. Cats were assigned to one of four treatment groups that received either high dose hulFN- $\alpha 2 a\left(10^{5} \mathrm{lU} / \mathrm{kg}\right.$ q24h; 12 cats), AZT (5 mg/kg q12h; 10 cats, both of these treatments $(12$ cats) or placebo (10 cats). All cats were treated for 6 weeks. Clinical variables, including stomatitis, and laboratory parameters, such as CD4 ${ }^{+}$and CD8 ${ }^{+}$counts and serum FeLV p 27 antigen concentration, were recorded throughout the treatment period. No significant difference among the groups was observed during the treatment period for any of the parameters. Aside from anaemia in one cat treated with AZT, no adverse effects were observed. It was not possible to demonstrate efficacy of hulFN- $\alpha 2 a$ or AZT alone or together in cats infected naturally with FeLV when given according to this regimen for 6 weeks; however, no notable side effects were detected.
\end{abstract}

Accepted: 11 December 2012

\section{Introduction}

Feline leukaemia virus (FeLV) is a $\gamma$-retrovirus that was first described in $1964 .{ }^{1}$ It occurs worldwide in domesticated cats and some related species. ${ }^{2,3}$ There are more studies published on antiviral treatment against FeLV infection than against any other virus infection in veterinary medicine, but, to date, no treatment described has resulted in cure or complete virus elimination. ${ }^{4}$

Human interferon- $\alpha 2 a$ (huIFN- $\alpha 2 a$ ) is commonly administered to cats, especially in countries such as the USA, where feline IFN (feIFN) is not available. In feline cell culture, replication of FeLV is inhibited by huIFN$\alpha 2 a$, and its efficacy is 10 times greater than that of feIFN. ${ }^{5}$ In cats infected experimentally with FeLV, 'lowdose' huIFN- $\alpha 2$ a prolonged the survival time and delayed development of FeLV-associated diseases when compared with non-treated animals, ${ }^{6}$ and survival time was longer than in cats treated with bovine (boIFN- $\beta$ ). ${ }^{7}$
The compound 3 '-azido-2', $3^{\prime}$-dideoxythymidine (zidovudine, AZT) has been used as an antiviral drug for patients infected with human immunodeficiency virus $(\mathrm{HIV}),{ }^{8}$ as well as for cats infected with FeLV. It also inhibits FeLV replication in vitro. ${ }^{9}$ In experimentallyinfected cats, development of FeLV-associated lymphomas was delayed in AZT-treated cats compared with non-treated cats. ${ }^{10}$ Cats infected naturally with FeLV and

\footnotetext{
${ }^{1}$ Clinic of Small Animal Medicine, LMU University of Munich, Germany

${ }^{2}$ Clinical Laboratory, Vetsuisse Faculty, University of Zurich, Switzerland
}

\section{Corresponding author:}

Bianca Stuetzer Dr med vet, Clinic of Small Animal Medicine, LMU University of Munich, Veterinaerstr. 13, 80539 Munich, Germany Email: stuetzer@|mu.de 
Table 1 Scoring of clinically evident stomatitis ( $0=$ no clinical sign; $100=$ most severe signs). The final score represents grades for intensity (from $0=$ no clinical sign to $10=$ most severe signs) and extent (from $0=$ no clinical sign to $10=$ most severe signs) of pharyngitis, gingivitis and glossitis. The product of the extent and intensity score for each of the three regions was summed and divided by three

\section{Stomatitis (score 0 to 100 )}

Pharyngitis

Gingivitis

Glossitis

Status of stomatitis
Intensity $(0-10) \times$ extent $(0-10)$

Intensity $(0-10) \times$ extent $(0-10)$

Intensity $(0-10) \times$ extent $(0-10)$

$($ Stomatitis + gingivitis + glossitis $) / 3$ treated with AZT for 3 weeks had significant improvement in their stomatitis. ${ }^{11}$

The combination of 'high-dose' huIFN- $\alpha$ and AZT seemed to be particularly promising. In a placebocontrolled study, prophylactic treatment of cats infected experimentally with FeLV using a combination of AZT and huIFN- $\alpha 2$ a prevented development of persistent viraemia. ${ }^{12}$ In addition, initiation of treatment with AZT and huIFN- $\alpha 2 \mathrm{a}$ in cats infected experimentally with FeLV with high levels of persistent antigenaemia, but prior to development of clinical signs, resulted in a significant decrease in FeLV p27 antigen levels 2 weeks after initiation of therapy.13 Therefore, the combination of AZT and huIFN- $\alpha 2 \mathrm{a}$ was clearly effective under experimental conditions. Thus, the aim of this study was to evaluate the efficacy of huIFN- $\alpha 2 a$ and AZT in cats infected naturally with FeLV. Specifically, clinical signs, changes in $\mathrm{CD}^{+}$ and $\mathrm{CD}^{+}$counts, and serum FeLV p 27 antigen concentrations were assessed. The primary hypothesis of the study was that FeLV antigen concentration would decrease by at least $60 \%$ within 6 weeks of treatment in naturally-infected cats. The secondary study hypothesis was that stomatitis would clinically improve within the same time period.

\section{Materials and methods}

\section{Study design}

The prospective study was designed as a placebo-controlled double-blinded clinical trial. It fulfilled the general German guidelines for prospective studies with appropriate owner consent. Forty-four cats were included and assigned randomly to one of four treatment groups. All cats stayed in the hospital during the 6-week treatment period. Cats in each group received one capsule PO q12h containing either AZT $(5 \mathrm{mg} / \mathrm{kg})$ (Retrovir; Glaxo Wellcome) or placebo (mannitol and aerosil), as well as one syringe SC q24h containing either huIFN- $\alpha 2 \mathrm{a}\left(10^{5} \mathrm{IU} / \mathrm{kg}\right)$ (Roferin-A Roche; Hoffmann-La Roche) or placebo (sterile physiologic saline, $0.9 \%$ ). Twelve cats received only huIFN- $\alpha 2 a, 10$ cats received only AZT, 12 cats received both AZT and huIFN- $\alpha 2 a$, and 10 cats received only placebo.

\section{Feline patients}

A total of 44 client-owned feline patients of the Clinic of Small Animal Medicine, LMU University of Munich, Germany, were included. Ten $(23 \%)$ were female intact, $11(25 \%)$ were female spayed, one $(2 \%)$ was male intact and $22(50 \%)$ were male neutered. The age of 38 of the 44 cats was known; these cats were between 1 and 10 years of age (median 4.3 years). Cats were eligible to enter the study if they had two positive FeLV test results [feline immunodeficiency virus (FIV)/FeLV combination test kit; Feline Leukemia Virus Antigen/Feline Immunodeficiency Virus Antibody Test Kit; Idexx]. Cats that tested positive for FIV, cats in moribund condition and cats showing aggressive behaviour were excluded.

\section{Variables investigated}

Variables investigated throughout the study to detect efficacy and adverse effects included clinical signs, laboratory parameters, $\mathrm{CD}_{4}^{+}$and $\mathrm{CD} 8{ }^{+}$counts and the $\mathrm{CD} 4 /$ CD8 ratio, and a quantitative measurement of FeLV p27 antigen. For the parameter stomatitis, a numerical scoring system from 0 (no clinical sign) to 100 (most severe signs) was designed. Intensity and extent of pharyngitis, gingivitis and glossitis were assessed and multiplied (Table 1).

Every week, haematological parameters were investigated in order to detect adverse effects. A complete blood count was measured using an automatic analyser (CellDyn 3500 R; Abbott Diagnostics); haematocrit was evaluated manually using a micro-haematocrit centrifuge (Hettich). A serum biochemistry profile was performed every 3 weeks with an automatic analyser (Hitachi 717 Autoanalyzer; Boehringer), a flame photometer (Effox 5053; Eppendorf) and a chloride analyser (Corning 925; Corning). The $\mathrm{CD}^{+}$and $\mathrm{CD} 8{ }^{+}$cell counts and the CD4/ CD8 ratio, as well as FeLV p27 antigen levels, were determined every 3 weeks.

\section{CD4+ and CD8+ cell counts}

To evaluate T-lymphocyte subsets, lymphocytes were stained for cell surface expression of CD3, CD4 and CD8 as described previously, ${ }^{14}$ and counted using a 
Table 2 Changes in variables during the treatment period. Numbers indicate the median difference between end and beginning of treatment, presented as \% of the values at beginning of treatment

\begin{tabular}{|c|c|c|c|c|c|}
\hline Parameter & hulFN- $\alpha 2 a$ & AZT & hulFN- $\alpha 2 \mathrm{a} / \mathrm{AZT}$ & Placebo & $P^{\star}$ \\
\hline Stomatitis & -4.8 & 0.6 & -8.7 & 1.1 & 0.080 \\
\hline Haemoglobin (g/dl) & -4.5 & -9.9 & -16.6 & -5.7 & 0.265 \\
\hline Haematocrit (\%) & -1.0 & -3.1 & -4.9 & -1.6 & 0.703 \\
\hline 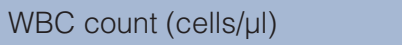 & 2.8 & -5.2 & -4.7 & -4.0 & 0.893 \\
\hline Relative CD4 count (\%) & 3.8 & 2.6 & 4.1 & -4.9 & 0.444 \\
\hline Relative CD8 count (\%) & -0.8 & -1.6 & 2.4 & 2.2 & 0.443 \\
\hline Absolute CD4 count (cells/ $/ \mathrm{l})$ & -2.0 & -12.3 & 106.2 & 5.6 & 0.335 \\
\hline Absolute CD8 count (cells/ul) & -33.0 & -22.5 & 96.7 & 63.7 & 0.089 \\
\hline CD4/CD8 ratio & 23.1 & 66.7 & -11.8 & -18.2 & 0.192 \\
\hline FeLV p27 antigen & 0.3 & -3.6 & -1.5 & 1.2 & 0.988 \\
\hline
\end{tabular}

${ }^{*}$ Comparison between groups by Kruskall-Wallis test

$\mathrm{WBC}=$ white blood cell; FeLV = feline leukaemia virus; hulFN- $\alpha 2 \mathrm{a}=$ human interferon- $\alpha 2 \mathrm{a}$

fluorescence-activated cell sorter (Becton Dickinson). In general, 30,000 cells were measured and analysed with two different software programs (Cell Quest version 1.1.1 and Macintosh Quadra 650). The absolute CD4 ${ }^{+}$ and $\mathrm{CD}^{+}$cell count was measured, and the CD4/CD8 ratio was calculated.

\section{FeLV-p27-Antigen ELISA}

Quantitative determination of FeLV p27 antigen was performed as described previously using an ELISA for quantification of free serum p27 antigen in relation to a defined positive control. ${ }^{15}$

\section{Statistical evaluation}

Prior to the study, a power analysis was conducted to determine the number of cats needed in each group so as to detect significant differences among the treatment groups, and between the treatment groups and the placebo group. Using data from the pilot study by Zeidner et al, ${ }^{12}$ assuming a SD in antigen decrease (80\%) of 400 $\mathrm{ng} / \mathrm{ml}$, a power of $90 \%$ and $\alpha=0.05$, at least 10 cats per group were calculated to be necessary to detect an antigen decrease of at least $60 \%$ in naturally-infected cats. This percentage decrease was chosen as it was considered likely to be clinically relevant. Thus, based on the power analysis, 10-12 cats per group were included in the study.

Stomatitis score, all laboratory parameters, lymphocyte subsets and FeLV p 27 antigen concentrations were all compared among treatment groups using commercial software (SPSS). Change over time was determined by calculating the difference between the mean values at the beginning and end of therapy. The Kruskall-Wallis test was used to analyse differences among groups. The Wilcoxon test was used to compare each drug group with the placebo group. For all analyses a $P$-value $<0.05$ was considered significant.

\section{Results}

\section{Efficacy of drugs}

All cats were still alive at the end of the study. Stomatitis was the most frequently observed clinical sign (37/44 cats; $81.8 \%$ ) and was the only clinical variable statistically compared among groups. No significant difference in stomatitis score was detected among treatment groups (Table 2).

Likewise, no significant differences among groups were detected for any immunological parameters (ie, $\mathrm{CD}^{+}$and $\mathrm{CD}^{+}$counts, and CD4/CD8 ratio). However, in cats treated with both AZT and huIFN- $\alpha 2 \mathrm{a}$, median absolute $\mathrm{CD}^{+}{ }^{+}$count increased by more than $100 \%$ (Table $2)$, whereas no significant increase was seen in the placebo group.

No significant differences in the development of the FeLV p27 antigen concentrations were observed among the groups.

\section{Adverse effects}

No significant differences in haematological or biochemistry parameters were detected among treatment groups. Haematocrit and haemoglobin concentration of only one cat treated with AZT decreased to below the reference interval during the treatment period.

\section{Discussion}

Although treatment of cats infected experimentally with FeLV with huIFN- $\alpha$ in combination with AZT is effective, ${ }^{12,13}$ treatment of naturally-infected cats in the present study using the same drugs was not effective. This was despite application of the same huIFN- $\alpha$ concentration $\left(10^{5} \mathrm{IU} / \mathrm{kg}\right)$ used in one of the successful experimental studies. ${ }^{13}$ Zeidner et al $^{13}$ prophylactically treated cats infected experimentally with FeLV using a combination of AZT and IFN- $\alpha 2 \mathrm{a}$, and could prevent the development of persistent viraemia. ${ }^{12}$ In another study evaluating the 
therapeutic effect of IFN- $\alpha 2 a$ and AZT used alone or in combination to treat experimentally-infected FeLV presymptomatic cats with established FeLV infection and persistent antigenaemia, Zeidner et $\mathrm{al}^{13}$ showed that treatment resulted in a significant decrease in circulating FeLV p27 antigen. On the contrary, in the present study, neither AZT nor IFN- $\alpha 2 a$, nor the combination of the compounds, resulted in a significant decrease in FeLV p27 antigenaemia.

When given alone in other studies, AZT was also not very effective in experimentally- or naturally-infected cats. ${ }^{11,13,16}$ Only when given prophylactically (before experimental infection), AZT had some effect and could prevent development of persistent viraemia.16,17 Although it was not to be expected that AZT alone would have a notable effect in naturally-infected FeLV cats, the combination of AZT and IFN- $\alpha 2 a$ was considered a promising approach. ${ }^{12}$ However, combination treatment in the present study was not associated with a statistically significant decrease in FeLV p27 antigen or improvement of clinical, laboratory or immunological variables. There are several possible reasons for these different results among studies. First, the difference between experimental and natural infection might be important. It is possible that experimentally-infected cats received treatment at an earlier stage of infection than did naturally-infected cats. In the early phase of infection, FeLV is potentially more vulnerable to antiviral drug effects than it is in cats that are already infected for a longer time, which is likely the case in naturallyinfected cats. During early viraemia, many cats are able to overcome viraemia and become FeLV antigen negative. ${ }^{4}$ Zeidner et $\mathrm{al}^{13}$ started treatment 3 weeks after infection, at a time point when, in many cats, a spontaneous decrease in p27 antigen is possible, even without treatment. However, once FeLV infection is established, as was likely in naturally-infected cats assessed in the present study, it might be much more difficult to influence viraemia and antigen concentration. Furthermore, in the studies of Zeidner et al, ${ }^{12,13}$ the cats were infected with one specific FeLV isolate (FeLV-FAIDS 61E/C) ${ }^{13}$ that might have been more susceptible to treatment, whereas cats in the present study were infected naturally with unknown, but likely different, FeLV strains. Another explanation might be the different dosage of AZT used in the present study $(5 \mathrm{mg} / \mathrm{kg}$ ) compared with the regimen of Zeidner et al $(20 \mathrm{mg} / \mathrm{kg}){ }^{13}$ This lower dosage was chosen in order to lower the risk of development of anaemia. Severe anaemia develops during treatment of cats infected naturally with FeLV at AZT dosages of $10 \mathrm{mg} / \mathrm{kg} .{ }^{11}$ Thus, a rather cautious approach was chosen in the present study. The huIFN$\alpha 2 \mathrm{a}$ dosage of $10^{5} \mathrm{IU} / \mathrm{kg}$ q24h used in the present study equates to the middle dosage used in one of the studies by Zeidner et $\mathrm{al}^{13}$ and was chosen in the present study because a higher dosage in cats would be expected to stimulate development of neutralising antibodies against the heterologous interferon within 3 weeks.

None of the drugs used in the present study had a significant effect on the most frequently observed clinical sign of stomatitis. This is in contrast to several placebo-controlled trials, in which treatment with AZT improved stomatitis in naturally-infected FIV cats. ${ }^{18-20}$ It is possible that AZT is not as effective against FeLV as it is against FIV because AZT is a drug designed for use in humans infected with HIV, and HIV and FIV reverse transcriptases are more similar to each other than are the enzymes of HIV and FeLV. ${ }^{21}$

Also, no significant effect of treatment was observed on $\mathrm{CD}^{+}$counts, $\mathrm{CD} 8{ }^{+}$counts or the $\mathrm{CD} 4 / \mathrm{CD} 8$ ratio in any group. These values might not be such valuable parameters for assessing immune function in cats infected with FeLV because, in contrast to FIV-infected cats, CD4 ${ }^{+}$ cells are not selectively destroyed in FeLV-infected cats. ${ }^{11}$

Surprisingly, in the present study administration of AZT of huIFN- $\alpha 2$ a alone or in combination was not associated with any adverse effects in most of the cats. By contrast, Zeidner et al ${ }^{12,13}$ observed adverse effects, such as anorexia and weight loss, in cats receiving huIFN- $\alpha 2 a$. In another study, tissue irritation and necrosis were described after subcutaneous injection of huIFN- $\alpha 2 a .{ }^{22}$ In addition, AZT can lead to anaemia and neutropenia due to a bone marrow depression. $13,19,23,24$ In the present study, a slight decrease in haematocrit and haemoglobin concentration was noted in all study groups, including the placebo group - a fact that could be explained by the frequent (once-weekly) blood collection throughout the course of the study. However, the amount of blood required for the laboratory tests reported in the article should not push most cats into anaemia, especially with the generous splenic reserves of erythrocytes in cats. Despite this general trend, only one cat (receiving AZT alone) developed haematocrit values and haemoglobin concentration below the reference interval. Thus, AZT may be associated with anaemia, but only in individual cats.

One limitation of the present study was the rather short treatment period (6 weeks). It is possible that changes in some variables would have been more pronounced and a significant effect might have been observed if treatment was continued for a longer period. However, this was not possible because IFN- $\alpha 2$ a-treated animals tend to develop neutralising antibodies to IFN- $\alpha 2 a$ within about 7 weeks of treatment depending on the dose administered..$^{13}$ Another limitation of the present study was that the duration of FeLV infection prior to therapy was not known. This likely resulted in a heterogeneous study population with respect to infection status.

Although group size in the present study was determined using power analysis prior to the study, this required estimation of a number of poorly understood 
variables, and it is possible that the failure to detect significant differences among the study groups might have resulted from insufficient cat numbers studied rather than to an actual lack of effect. Finally, provirus load was not quantified in the present study. It is possible that provirus load may have differed among treatment groups; however, changes in provirus load are typically seen in feline patients on extended treatment regimens, whereas antigen detection is considered a valuable tool to determine viral replication rate and therefore better for detecting earlier differences.

\section{Conclusions}

It can be concluded from the present study that although efficacy of 'high-dose' huIFN- $\alpha 2 a$ and AZT has been demonstrated in cats infected experimentally with FeLV, no significant effect of these compounds (as administered here) could be demonstrated in cats naturally infected with FeLV. However, it should also be noted that no adverse effects were seen. It is possible that efficacy could be observed if these drugs were administered at a very early stage of FeLV infection.

Acknowledgements The authors thank the Clinic of Small Animal Medicine of the LMU University of Munich for providing financial support of the study and Professor Dr David Maggs for carefully reviewing the manuscript.

Funding This research received no grant from any funding agency in the public, commercial, or not-for-profit sectors.

Conflict of interest The authors do not have any potential conflicts of interest to declare.

\section{References}

1 Jarrett WF, Crawford EM, Martin WB and Davie F. A Viruslike particle associated with leukemia (lymphosarcoma). Nature 1964; 202: 567-569.

2 Hoover EA and Mullins JI. Feline leukemia virus infection and diseases. J Am Vet Med Assoc 1991; 199: 1287-1297.

3 Leutenegger CM, Hofmann-Lehmann R, Riols C, et al. Viral infections in free-living populations of the European wildcat. J Wildl Dis 1999; 35: 678-686.

4 Levy J, Crawford C, Hartmann K, et al. 2008 American Association of Feline Practitioners' feline retrovirus management guidelines. J Feline Med Surg 2008; 10: 300-316.

5 Jameson $\mathrm{P}$ and Essex M. Inhibition of feline leukemia virus replication by human leukocyte interferon. Antiviral Res 1983; 3: 115-120.

6 Cummins JM, Tompkins MB, Olsen RG, et al. Oral use of human alpha interferon in cats. J Biol Response Mod 1988; 7: 513-523.

7 Weiss RC, Cummins JM and Richards AB. Low-dose orally administered alpha interferon treatment for feline leukemia virus infection. J Am Vet Med Assoc 1991; 199: 1477-1481.

8 Fischl MA, Richman DD, Grieco MH, et al. The efficacy of azidothymidine (AZT) in the treatment of patients with
AIDS and AIDS-related complex. A double-blind, placebo-controlled trial. New Engl J Med 1987; 317: 185-191.

9 Tateyama S, Priosoeryanto BP, Yamaguchi R, et al. In vitro growth inhibition activities of recombinant feline interferon on all lines derived from canine tumours. Res Vet Sci 1995; 59: 275-277.

10 Nelson P, Sellon R, Novotney C, et al. Therapeutic effects of diethylcarbamazine and 3 '-azido-3'-deoxythymidine on feline leukemia virus lymphoma formation. Vet Immunol Immunopathol 1995; 46: 181-194.

11 Hartmann K, Donath A, Beer B, et al. Use of two virustatica (AZT, PMEA) in the treatment of FIV and of FeLV seropositive cats with clinical symptoms. Vet Immunol Immunopathol 1992; 35: 167-175.

12 Zeidner NS, Rose LM, Mathiason-DuBard CK, et al. Zidovudine in combination with alpha interferon and interleukin-2 as prophylactic therapy for FeLV-induced immunodeficiency syndrome (FeLV-FAIDS). J Acquir Immune Defic Syndr 1990; 3: 787-796.

13 Zeidner NS, Myles MH, Mathiason-DuBard CK, et al. Alpha interferon (2b) in combination with zidovudine for the treatment of presymptomatic feline leukemia virusinduced immunodeficiency syndrome. Antimicrob Agents Chemother 1990; 34: 1749-1756.

14 Hoffmann-Fezer G, Thum I, Herbold M, et al. T-helper and $\mathrm{T}$-suppressor lymphocyte subpopulations in the peripheral blood of spontaneously FIV-positive cats. Tierarztliche Praxis 1991; 19: 682-686 [in German].

15 Lutz H, Pedersen NC and Theilen GH. Course of feline leukemia virus infection and its detection by enzymelinked immunosorbent assay and monoclonal antibodies. Am J Vet Res 1983; 44: 2054-2059.

16 Tavares L, Roneker C, Johnston K, et al. 3'-Azido-3'-deoxythymidine in feline leukemia virus-infected cats: a model for therapy and prophylaxis of AIDS. Cancer Res 1987; 47: 3190-3194.

17 Mathes LE, Polas PJ, Hayes KA, et al. Pre- and postexposure chemoprophylaxis: evidence that 3 '-azido-3'-dideoxythymidine inhibits feline leukemia virus disease by a drug-induced vaccine response. Antimicrob Agents Chemother 1992; 36: 2715-2721.

18 Hartmann K. AZT in the treatment of feline immunodeficiency virus infection: part 1. Feline Pract 1995; 23: 16-21.

19 Hartmann K. AZT in the treatment of feline immunodeficiency virus infection: part 2 . Feline Pract 1995; 23: 13-20.

20 Hartmann K. Feline immunodeficiency virus infection. Brit Vet J 1998; 155: 123-137.

21 North T, North G and Pedersen N. Feline immunodeficiency virus, a model for reverse transcriptase-targeted chemotherapy for acquired immune deficiency syndrome. Antimicrob Agents Chemother 1989; 33: 915-919.

22 Zimmermann C, Walther EU, Goebels N, et al. Interferon beta-1b for treatment of secondary chronic progressive multiple sclerosis. Der Nervenarzt 1999; 70: 759-763 [in German].

23 McLeod GX and Hammer SM. Zidovudine: five years later. Ann Intern Med 1992; 117: 487-501.

24 Haschek WM, Weigel RM, Scherba G, et al. Zidovudine toxicity to cats infected with feline leukemia virus. Fundam Appl Toxicol 1990; 14: 764-775. 